Journal of

Cardiology and Vascular Medicine

\title{
Efficacy and Safety of Extended Duration Use of Anticoagulants in Acutely Ill Medical Patients
}

\section{Ali Hamoudi $\mathrm{MD}^{1, *}$, VianTaqi $\mathrm{MD}^{2}$}

Rosalind Franklin University, North Chicago, IL, USA

University of Baghdad, Baghdad, Iraq

${ }^{\star}$ Corresponding author: Ali Hamoudi MD, Rosalind Franklin University, North Chicago, IL, USA; E-mail:ali.hamoudi@rosalindfranklin.edu

Received Date: April 06, 2019 Accepted Date: May 08, 2019 Published Date: May 09, 2019

Citation: Ali Hamoudi (2019) Efficacy and Safety of Extended Duration Use of Anticoagulants in Acutely Ill Medical Patients. J Cardio Vasc Med 5: 1-4.

\begin{abstract}
Venous thromboembolism (VTE) is a common serious disease with significant morbidity and mortality in both hospitalized and acutely ill medical patients. Anticoagulant is now the standard of care for inpatients to prevent as well as treat thrombosis. The high risk of recurrence after the initial episode makes further intervention mandatory.

One of the difficulties that face clinicians is anticoagulant treatment associated with an increased risk for bleeding complications. Another challenge is the optimal duration to protect from another attack which remains unclear.

Understanding that persistent risk factors like (malignancy, hypercoagulable disorders, and idiopathic DVT) had a 2- to 3-fold higher risk of recurrence than those with transient risk factors like major surgery and lower-limb fracture is another part of the equation.

Multiple Randomized, controlled trials of parenteral extended duration anticoagulants versus standard care or placebo in hospitalized medical patients have shown a reduction of more than $50 \%$ in the rate of venous thromboembolism, including fatal pulmonary embolism. This review is focusing on the efficacy and safety of extended duration anticoagulant use.
\end{abstract}

Keywords: Venous thromboembolism; Deep venous thrombosis; Anticoagulant; Thrombosis; Pulmonary embolism

(C)2019 The Authors. Published by the JScholar under the terms of the Creative Commons Attribution License http://creativecommons.org/licenses/ by/3.0/, which permits unrestricted use, provided the original author and source are credited. 


\section{Introduction}

Venous thromboembolism (VTE) is a common serious disease with significant morbidity and mortality in both hospitalized and acutely ill medical patients. Anticoagulant is now the standard of care for inpatients to prevent as well as treat thrombosis. The high risk of recurrence after the initial episode makes further intervention mandatory. It's well known that short-term thromboembolic prophylaxis with anticoagulants for 1-2 weeks is effective in reducing the recurrence rate of venous thromboembolism in both medical and surgical patients [1-3].

\section{Materials \& Methods}

Studies have shown that there is an increased risk of VTE after hospital discharge especially during the first 30 days [4]. Below are few of well-established clinical trials that used to assess the efficacy and safety of extended duration thromboprophylaxis after leaving the hospital:

The EXCLAIM study (Extended Clinical prophylaxis in Acutely ill Medical patients) involved 4,726 acutely ill medical patients with recently reduced mobility, was designed to compare the efficacy and safety of extended-duration thromboprophylaxis. The study compared using $40 \mathrm{mg}$ once daily enoxaparin (38 \pm 4 days) after the standard regimen for enoxaparin (40 mg once daily for $10 \pm 4$ days) with the placebo after the same standard regimen. The Main Objective was to document that extended use of Enoxaparin thromboprophylaxis is an effective intervention for patients with recent VTE after being discharged out of the hospital.

Another Clinical trial is MAGELLAN study (Multicenter, Randomized, Parallel Group Efficacy and safety) involved 8428 patients for prevention of VTE in hospitalized acutely Ill medical patients that was designed to assess the efficacy and safety of extended use of oral rivaroxaban $10 \mathrm{mg}$ for $35 \pm 4$ days and subcutaneous enoxaparin placebo compared with standard-duration (10 \pm 4 days) of subcutaneous enoxaparin $40 \mathrm{mg}$ for $10 \pm 4$ days and oral rivaroxaban placebo for $35 \pm 4$ days. It also used to evaluate the role of extended-duration of rivaroxaban for 5 weeks of thromboprophylaxis in acutely ill medical patients.

One of the recent trials that were published in 2016 is the APEX trial. According to APEX, A total of 7513 patients who were hospitalized for acute medical illnesses were assessed for extended use of anticoagulant in term of efficacy and safety. Two groups were randomly assigned to receive subcutaneous enoxaparin (at a dose of $40 \mathrm{mg}$ once daily) for $10 \pm 4$ days plus oral betrixaban placebo for 35 to 42 days or subcutaneous enoxaparin placebo for $10 \pm 4$ days plus oral betrixaban (at a dose of $80 \mathrm{mg}$ once daily) for 35 to 42 days.
The aim of this review is to focus on APEX trial and compare the results of it with Magellan and Exclaim trials results in term of extended VTE prophylaxis. According to APEX trial, Patients were eligible if they were 40 years of age or older, had been hospitalized for less than 96 hours for a specific illness (heart failure, respiratory failure, infectious disease, rheumatic disease, or ischemic stroke), with reduced mobility and specific risk factors for venous thromboembolism $[5,6]$.

The study performed sequential analysis by establishing two cohorts within the overall study population: patients with an elevated d-dimer level (cohort 1), patients with an elevated d-dimer level or an age of at least 75 years (cohort 2).

The primary efficacy endpoint just like the other studies was VTE, which defined as the composite of symptomatic or asymptomatic proximal DVT, symptomatic PE, or fatal $\mathrm{PE}$, during the double-blind period of extended prophylaxis. While the primary safety endpoint was the incidence of major and clinically relevant non-major hemorrhagic complications during the double-blind treatment period.

\section{Results}

EXCLAIM study demonstrated that extended-duration use of enoxaparin significantly reduced the overall incidence of VTE in acutely ill medical patients with reduced mobility, but with a significant increase in the incidence of major bleeding. The absolute risk difference for the reduction in the incidence of VTE was $-1.53 \%$ [95.8\% confidence interval $[\mathrm{CI}],-2.54$ to $-0.52 \%$ ] and the absolute risk difference for the increase of the risk of bleeding was $0.51 \%$ [95\% CI, 0.12 to $0.89 \%]$ ) [7].

For MAGELLAN study, By day 10, harm an event of the primary efficacy outcome or major or clinically relevant non major bleeding had occurred in $(6.6 \%)$ in the rivaroxaban group, as compared with (4.6\%) in the enoxaparin group (relative risk, 1.44; 95\% CI, 1.18 to $1.77 ; \mathrm{P}<0.001$ ). By day 35 , an event of this composite outcome had occurred in $(9.4 \%)$ in the group that received extended-duration rivaroxaban, as compared with $(7.8 \%)$ in the group that received enoxaparin followed by placebo (relative risk, $1.21 ; 95 \% \mathrm{CI}, 1.03$ to 1.43 ; $\mathrm{P}=0.02)[8]$.

For APEX trial, In cohort 1 , the primary efficacy outcome occurred in $6.9 \%$ of the betrixaban group and $8.5 \%$ of the enoxaparin group (relative risk in the betrixaban group, 0.81; $95 \%$ confidence interval [CI], 0.65 to $1.00 ; \mathrm{P}=0.054$ ). In cohort 2 , the primary efficacy outcome occurred in $5.6 \%$ of the betrixaban group and $7.1 \%$ of the enoxaparin group (relative risk, 0.80 ; $95 \% \mathrm{CI}, 0.66$ to $0.98 ; \mathrm{P}=0.03$ ). In the overall population, the primary efficacy outcome occurred in $5.3 \%$ and $7.0 \%$ of the patients, respectively (relative risk, 0.76 ; $95 \%$ CI, 0.63 to 0.92 ; $\mathrm{P}=0.006)$. 
In term of safety In the overall population, major bleeding occurred in $0.7 \%$ of the betrixaban group and $0.6 \%$ of the enoxaparin group (relative risk, $1.19 ; 95 \% \mathrm{CI}, 0.67$ to 2.12 ; $\mathrm{P}=0.55$ ) while clinically relevant non major bleeding occurred in $3.1 \%$ of the betrixaban group and $1.6 \%$ of the enoxaparin group (relative risk, $1.97 ; 95 \% \mathrm{CI}, 1.44$ to 2.68 ; $\mathrm{P}<0.001$.

Based on Apex trial, patients who have elevated d-dimer level or an age of 75 years or more may have the greater risk of venous thromboembolism and at the same time a greater benefit of extended-duration antithrombotic prophylaxis. These expectations were based on data for similar patients who were enrolled in the MAGELLAN trial of rivaroxaban.

\section{Discussion}

Although venous thromboembolism (VTE) is primarily a postoperative complication in surgical patients, it has also been reported up to $30 \%$ of hospitalized medical patients with an incidence comparable to that of surgical patients.

The high risk of venous thromboembolism after the hospital discharge following acute medical illnesses or postsurgical patients requires greater attention to diagnose and treat. Although venography is the gold standard for DVT diagnosis especially asymptomatic one, compression ultrasound has been used more commonly in these studies. One of the limitations to the APEX trial is Compression U/S not performed in up to $15 \%$ of patients.

In Magellan study, the efficacy of standard-duration rivaroxaban was similar to that of enoxaparin, whereas the efficacy of extended-duration rivaroxaban $35 \pm 4$ days was superior to that of enoxaparin administered for the standard duration (10 \pm 4 days). However, rivaroxaban was associated with an increased risk of major and clinically relevant non-major bleeding.

The Exclaim study reported that extended-duration enoxaparin $(10+28$ days enoxaparin prophylaxis) reduced the risk of VTE compared to standard enoxaparin ( $10 \pm 4$ days). The most notable reduction in risk of VTE was noticed in Women, older patients ( $>75$ years), and sedentary patients. There is a risk of increased major bleeding complications in the extended duration thromboprophylaxis arm compared to the standard arm. This subanalysis assessed the risk of VTE and bleeding with extended-duration enoxaparin prophylaxis in patients with acute ischemic stroke $[7,9]$.

With Apex trial it did not show any statistical significance of betrixaban when compared to Enoxaparin (p-value was less than 0.05 ) in term of primary efficacy so all subsequent efficacy outcomes were considered exploratory. However, Oral betrixaban use can be extended after discharge to reduce the rate of venous thromboembolism among patients who are categorized according to their admission diagnoses and predefined risk factors.

Betrixaban was also associated with a low frequency of major bleeding and fatal bleeding. Unlike enoxaparin in the EXCLAIM trial, and rivaroxaban in the MAGELLAN trial, the use of extended-duration betrixaban in APEX trial was not associated with significantly more major bleeding than standard-duration use of enoxaparin. There was significantly more clinically relevant non-major bleeding. Intracranial bleeding was infrequent in the two groups, but the rate was lower in the betrixaban group than in the enoxaparin group.

\section{Conclusions}

Despite extended prophylaxis for VTE is recommended in the post-operative surgical patients especially after orthopedic surgeries compared to the standard care, anticoagulant use in the medical setting is still challenging.

Extended duration anticoagulants use is recommended to prevent VTE in post-hospital discharge patients. This was established in both the standard and novel type anticoagulants. comparing the benefits of preventing further blood clots versus the risks of bleeding may favor use anticoagulant especially in patients with low risks of bleeding.

We recommended using VTE prophylaxis in a patient with well-established risk factors that may be aggravated by the hospital stay.

The optimum duration of anticoagulant use should be adjusted so it does not expose those patients to bleeding complications. It is reasonable to adjust the duration of anticoagulant use based on the complexity of the patients' medical condition and clinical sequels after the discharge. Further studies may be needed in the future for the optimum duration to give these anticoagulants. 


\section{References}

1. Alikhan R, Cohen AT (2009) Heparin for the prevention of venous thromboembolism in general medical patients (excluding stroke and myocardial infarction). Cochrane Database Syst Rev.

2. Dentali F, Douketis JD, Gianni M, Lim W, Crowther MA (2007) Meta-analysis: anticoagulant prophylaxis to prevent symptomatic venous thromboembolism in hospitalized medical patients. Ann Intern Med 146:278-288.

3. Collins R, Scrimgeour A, Yusuf S, Peto R (1998) Reduction in fatal pulmonary embolism and venous thrombosis by perioperative administration of subcutaneous heparin. Overview of results of randomized trials in general, orthopedic, and urologic surgery. N Engl J Med 318:1162-1173.

4. Amin AN, Varker H, Princic N, Lin J, Thompson S, Johnston S (2012) Duration of venous thromboembolism risk across a continuum in medically ill hospitalized patients. J Hosp Med 7:231-238.

5. Samama M-M (2000) An epidemiologic study of risk factors for deep vein thrombosis in medical outpatients: the Sirius study. Arch Intern Med 160:3415-3420.

6. Samama MM, Dahl OE, Quinlan DJ, Mismetti P, Rosencher N (2003) Quantification of risk factors for venous thromboembolism: a preliminary study for the development of a risk assessment tool. Haematologica. 88:1410-1421.

7. Hull RD, Schellong SM, Tapson VF (2010) Extendedduration venous thromboembolism prophylaxis in acutely ill medical patients with recently reduced mobility: a randomized trial 153: 8-18.

8. Alexander T. Cohen MD, Theodore E, et al. (2013) Rivaroxaban for Thromboprophylaxis in Acutely Ill Medical Patients. 368:513-523.

9. Samuel Z. Goldhaber M.D, Alain Leizorovicz M.D, Ajay K. Kakkar M.D, et al. Apixaban versus enoxaparin for thromboprophylaxis in medically ill patients.

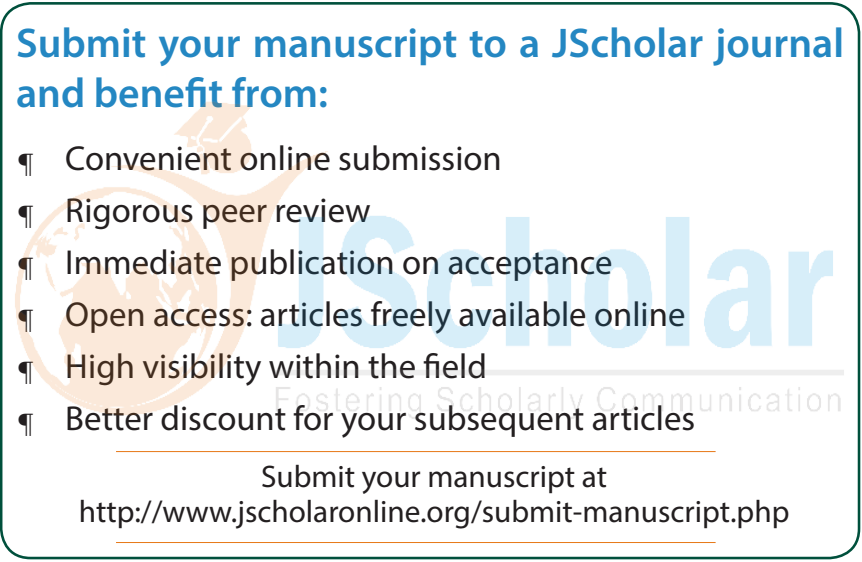

